\title{
Bedeutung von Mikrobiomen in Darm und Atemwegen für Therapien
}

Es mehren sich die Hinweise, dass die Atemwege von Patienten mit Asthma keineswegs steril sind, sondern eine bestimmte bakterielle Kolonisierung mit sogenannten Proteobakterien, insbesondere Haemophilus influenzae, Moraxella catarrhalis und Streptococcus pneumoniae aufweisen, wie das zuerst von Hilti et all. in 2010 bei Kindern und Erwachsenen mittels bronchoalveolärer Lavagen bzw. bronchialer Bürstungen gezeigt worden ist. Die Frage, ob diese Kolonisierung nun einfach ein Begleitphänomen von Asthma ist oder möglicherweise im kausalen Zusammenhang mit der Entwicklung dieser Erkrankung steht, wurde in der COPSAC-Studie untersucht. In dieser wurde gezeigt, dass die bakterielle Kolonisierung des NasenRachenraums im Alter von vier Wochen bereits der Entwicklung von Asthma bronchiale vorausging. Allerdings war dies ein unspezifischer Befund, da dieselbe Kolonisierung auch der Entwicklung von Pneumonie bzw. Bronchiolitis vorausging.

Die Frage stellt sich nun, welche Rolle das Umweltmikrobiom für das humane Mikrobiom spielt. Wir haben für diese Fragestellung die Bauernhofstudien zur Hand, die uns weitere Hinweise geben können. In der sogenannten PASTURE-Geburtskohorte haben wir das Vorkommen von Bakterien in Nicht-Bauern- und Bauernhäusern untersucht, und wir sehen ein unterschiedliches Vorkommen: in Bauernhäusern finden sich viele, aber in jeweils geringer Menge, Keime aus dem Kuhstall, in Nicht-Bauernhäusern hingegen befinden sich mehr Keime menschlichen Ursprungs.

Wenn man sich aber Keime ansieht, die in größeren Mengen vorkommen, dann überlappen sich die Verteilungen durchaus zwischen Bauern- und Nicht-Bauernhäusern. Wir haben deswegen einen Farm-Index gebildet, der Keime, die besonders stark mit Bauernhof assoziiert sind, widerspiegelt. Wenn diese Keime auch in Nicht-Bauernhäusern auftreten, sind sie auch mit dem Schutz vor Asthma assoziiert. Welche Keime das genau sind, ist zurzeit Gegenstand der Untersuchungen.
Die nächste Frage ist, ob diese Keime aus der Umwelt auch das menschliche Mikrobiom, entweder inhaliert über den NasenRachenraum oder aber über den Darm, prägen. Zunächst haben wir Nasen- und Rachenabstriche von Kindern von Bauernhöfen und Nicht-Bauernhöfen im Schulalter untersucht. Wir sehen im Rachen, dass Kinder, die auf dem Bauernhof leben, eine höhere Diversität haben, aber dass kein Zusammenhang mit Asthma bestand. Hingegen fanden wir das Nasenmikrobiom von wesentlich größerer Bedeutung. Da gab es auch mehr Diversität, wenn die Kinder auf einem Bauernhof aufgewachsen waren. Diese Diversität führte zu weniger Kolonisierung mit Moraxella und dadurch auch zu weniger Asthma.

Die Rolle des Darmmikrobioms haben wir uns in der PASTURE-Kohorte genauer angesehen, wo wir Proben im Alter von zwei und zwölf Monaten gesammelt haben. Wie andere auch, haben wir gesehen, dass sich die Zusammensetzung des Darmmikrobioms im ersten Lebensjahr erheblich entwickelt. Zunächst haben wir gezeigt, dass bereits im Alter von zwei Monaten ein Zusammenhang des Darmmikrobioms mit Asthmaentwicklung besteht, wobei Umweltfaktoren eine Rolle spielen. Es erfolgt eine signifikante Reifung des Darmmikrobioms im ersten Lebensjahr, und diese Reifung war mit Asthma assoziiert. Auch diese Reifung wurde von Umweltfaktoren beeinflusst und ist durch ein komplexes Netzwerk von verschiedenen Bakterien gekennzeichnet. Bakterien bilden Metabolite, die wahrscheinlich in der Vermittlung dieser Effekte eine wichtige Rolle spielen.

Die mir gestellte Frage, ob das Konsequenzen für die Therapie hat, kann ich derzeit verneinen. Es gibt keine Hinweise, dass eine antibiotische Behandlung für Asthma von Vorteil wäre. Vielmehr müssen wir über neue Wege der Supplementation oder der gezielten Eradikation nachdenken.

\section{Interessenkonflikt}

Frau Prof. von Mutius gibt Honorare der folgenden Firmen und Institutionen an: Pharmaventures, OM Pharma S. A., SpringerVerlag $\mathrm{GmbH}$, Elsevier $\mathrm{GmbH}$ sowie Elsevier Ltd., Peptinnovate Ltd., Turun Yliopisto, Tampereen Yliopisto, Helsingin Yliopisto, European Respiratory Society, Deutsche Pharmazeutische Gesellschaft e. V., Massachusetts Medical Society, Chinese University of Hongkong, Europäische Kommission, Boehringer Ingelheim International $\mathrm{GmbH}$, Universität Utrecht Fakultät für Veterinärmedizin, Universität Salzburg, Georg Thieme Verlag, Japanese Society of Pediatric Allergy and Clinical Immunology (JSPACI. Des Weiteren hat Frau Prof. von Mutius folgendes Patent angemeldet: „LU101064 - Barn dust extract for the prevention and treatment of diseases". Sie besitzt das Patent „EP2361632: Specific environmental bacteria for the protection from and/or the treatment of allergic, chronic inflammatory and/ or autoimmune disorders“, dessen Lizenzgebühren an die Firma ProtectImmun GmbH gehen. Das Patent „EP 1411 977: Composition containing bacterial antigens used for the prophylaxis and the treatment of allergic diseases" ist zugelassen auf die Firma Protectlmmun GmbH. Patent Nummer „EP1637147: Stable dust extract for allergy protection“ ist ebenfalls zugelassen auf die Protectlmmun $\mathrm{GmbH}$, ebenso Patent „EP 1964570: Pharmaceutical compound to protect against allergies and inflammatory diseases".

\section{Autorin}

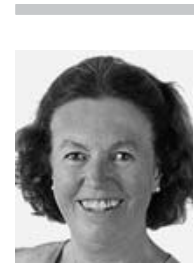

\section{Erika von Mutius}

Prof. Dr. med. Dr. h.c., Oberärztin der Klinik, Leiterin der Asthma- und Allergieambulanz, Leiterin des Instituts für Asthma- und Allergieprävention (IAP) am Helmholtz Zentrum München

\section{Korrespondenzadresse}

Prof. Dr. Dr. h.c. Erika von Mutius Kinderklinik und Kinderpoliklinik im Dr. von Haunerschen Kinderspital Klinikum der Universität München Lindwurmstraße 4 80337 München Deutschland erika.von.mutius@med.Imu.de 